\title{
LA RISA Y LOS ACTOS AMENAZANTES DE IMAGEN
}

\author{
Alexa Bolaños Carpio
}

\section{(c) $(1) \Theta(9)$}

Esta obra está bajo una licencia Creative Commons

Reconocimiento-No Comercial-Sin Obra Derivada 



\title{
LA RISA Y LOS ACTOS AMENAZANTES DE IMAGEN
}

\author{
LAUGHTER AND IMAGE'S MENACING ACTS
}

\section{Alexa Bolaños Carpio}

\begin{abstract}
RESUMEN
El artículo plantea que la risa funciona como una estrategia de cortesía cuando aparece en actos amenazantes de imagen. Los datos provienen de la grabación en video de interacción natural entre tres amigos cercanos. Se encontraron 81 episodios de risa con presencia de actos amenazantes de imagen, entre ellos: impropiedades conversacionales, enunciados comprometidos, críticas y desacuerdos. Los datos arrojados por el análisis pragmalingüístico evidencian que la risa presente en actos amenazantes de imagen cumple la función de ser una estrategia de cortesía para fortalecer la imagen social, mitigar un potencial ataque o reforzar una amenaza de imagen.

Palabras clave: risa, estrategia de cortesía, imagen social, actos amenazantes de imagen, pragmalingüística.
\end{abstract}

\begin{abstract}
This article examines laughter as a politeness strategy when it appears in face-threatening acts. The data comes from a video-recorded and naturally occurring interaction from the everyday context between three close friends. Eighty-one instances of laughter were found in face-threatening acts such as conversational inappropriateness, risky statements, criticism, and disagreement. The pragmalinguistic analysis reveals that the laughther appeared in face-threatening acts works as politeness strategy while strengthening social face, mitigating a potential attack or reinforcing a face threatening.

Key words: laughter, politeness strategy, social face, face-threatening acts, pragmalinguistics.
\end{abstract}

\section{Introducción}

La lingüística ha aportado conocimientos al estudio de la risa y sus investigaciones se centran en valorarla como un mecanismo con funciones específicas dentro de la conversación: mostrar vínculo y confianza entre los participantes (Bravo, 1996 y 1997; Caroll, 2002), salvar la imagen (Bravo, 2000), liberar la tensión del relato (Bravo, 2000) y regular el flujo de la

M.L. Alexa Bolaños Carpio. Universidad de Costa Rica. Profesora de la Sede del Atlántico. Costa Rica. Correo electrónico: alexa.bolanos@ucr.ac.cr

Recepción: 20- 01- 2015

Aceptación: 27- 01- 2015 
interacción (Cestero-Mancera, 1996; Devereux y Ginsburg, 2001; Bolaños, 2010b). Además, se indica que su presencia está ligada a la solidaridad entre los hablantes y no a eventos humorísticos (Provine, 1996; Robinson y Smith-Lovin, 2001; Carroll, 2002; Glenn, 2003; Bachorowski y Owren, 2004; Soilevuo-Grønnerød, 2004).

La razón de estudiar la risa desde la lingüística responde al hecho, planteado por Tusón (2002), de que conversar va más allá de las palabras "dichas". Se puede, entonces, inferir que el análisis de la conversación permite incluir dentro de su quehacer el estudio de otros elementos que no son, exclusivamente, verbales; es decir, podría incluirse la tos, los silencios, los ademanes, los gestos y la risa.

En el caso de este artículo se estudia la risa desde el análisis de la conversación, la pragmalinguiística y los estudios sobre estilos conversacionales (ver fundamento teórico). Para ello se presentan los resultados - asociados a los actos amenazantes de imagen- arrojados por una conversación entre amigos, todos hombres universitarios entre 20 y 30 años de edad (Bolaños, 2007). En los siguientes dos apartados se esbozarán la teoría y la metodología empleada para el tratamiento de datos.

\section{Fundamento teórico}

El marco teórico para un análisis integral de la risa, específicamente en conversaciones coloquiales entre personas con grados de amistad, debe contemplar tres apartados: el análisis de la conversación, la pragmalingüística y el estudio de los estilos conversacionales. La primera disciplina es como una "gran sombrilla" que cubre las dos restantes, pues es justamente en las conversaciones donde se presentan las temáticas de trabajo de las otras dos áreas; además, es una rama que posibilita contemplar tanto los aspectos verbales como los no verbales y, justamente, en estos últimos se incluye la risa. La pragmalingüística es necesaria para dar cuenta de la risa como una estrategia dentro de la interacción comunicativa, pues se analiza a la luz de nociones como el poder y la solidaridad en las relaciones sociales, el principio de cortesía, las máximas conversacionales, las implicaturas e ironías, la imagen pública de los hablantes, los actos de habla, el contexto y el co-texto, el sentido de no seriedad. La inclusión del estudio de los estilos conversacionales entre amigos permite comprender que el estilo de los participantes es un factor que puede afectar la aparición de episodios de risa (para mayores detalles cf. Bolaños, 2007).

Ahora bien, para comprender la risa debe empezarse por definirla: es un elemento no verbal constituido por una parte auditiva y otra facial (Bravo, 1997). Asimismo, es una reacción de todo el cuerpo, pues se observa un cambio en la postura de tensión-relajación. Cestero-Mancera (1996) define la risa como una actividad conversacional y un elemento paralingüístico también conversacional y plurifuncional, pues sirve de calificador de enunciados y de regulador de la interacción. Con esta última función concuerdan Devereux y Ginsburg (2001), quienes analizan la risa como un mecanismo para mantener o reestablecer el flujo de la conversación, y también Bolaños (2010b), quien considera la risa como un elemento que funciona en el nivel de la estructura conversacional.

Para efectos de este trabajo, un episodio de risa se entenderá como aquel donde "por lo menos una risa es emitida por el hablante" (Bravo, 2000, p. 141). Las risas, a su vez, pueden ser aisladas o compartidas. En el primer caso, será producida solamente por el emisor; en el segundo, involucra tanto al emisor como al destinatario, a quien se le ha atribuido el papel de cómplice del primero. 


\section{Metodología}

Los datos presentados en este artículo se desprenden del análisis realizado a una conversación caracterizada por ser coloquial, natural y entre personas con una relación de amistad. En la interacción participaron tres hombres: Miguel, 25 años; Ernesto, 21 años; y Joaquín, 23 años. Todos ellos son amigos y, a la vez, estudiantes universitarios. Para efectos de este estudio, la intervención de cada hablante será indicada mediante las tres primeras letras del nombre. Cabe reiterar que los nombres han sido cambiados.

La conversación se grabó en casete de audio y de vídeo para un mejor manejo y registro de los datos. A pesar de la presencia de la cámara y la grabadora, se considera con un alto grado de naturalidad debido a que no fue dirigida por la investigadora, ya que "the data are naturalistic in that no attempt was made to control how people talk or what they talk about on the recordings"3 (Glenn, 2003, p. 40). Sobre el mecanismo de obtención del corpus pesa el eterno dilema de la "paradoja del observador"; sin embargo, Duranti (2000) lo refuta al asegurar que los participantes no confeccionan su conducta o su lenguaje por el hecho de ser filmados, sino que tal conducta y lenguaje pertenecen a un repertorio de acciones independiente de la presencia de la cámara. Además, con el transcurrir de los minutos, los participantes de esta investigación se olvidaron de los aparatos y se observaron, mediante el lenguaje no verbal, más relajados y naturales.

La grabación tuvo una duración de dos horas; sin embargo, solo se utilizan cuarenta y cinco minutos. Tal decisión obedece a que, tanto en los primeros minutos como en los últimos, la investigadora estuvo presente y los participantes debían cambiar manualmente el casete. Este último factor generaba la atención hacia el equipo de grabación, por lo cual no se utilizaron los primeros minutos de cada lado del casete de audio. Se utilizaron quince minutos del primer lado del casete de audio y treinta minutos del segundo lado. El corpus de trabajo consta de 130 emisiones de risa presentadas de manera aislada y compartida, de los cuales 1 corresponde a desacuerdos, 23 a enunciados comprometidos, 12 a críticas y 45 a impropiedades conversacionales (Bolaños, 2007). No obstante, para efectos de este artículo solo se presentan los episodios más significativos sobre actos amenazantes de imagen.

Para la transcripción del corpus de trabajo, se emplearon las convenciones propuestas por Briz (1998) en el proyecto del grupo Val.Es.Co. (Valencia Español Coloquial); sin embargo, con el fin de adaptarlas al análisis de la risa, fue necesario efectuarle algunas modificaciones (ver anexo).

\section{Análisis}

En la conversación coloquial entre amigos, la risa puede aparecer en enunciados que presentan actos amenazantes de imagen, entre ellos: impropiedades conversacionales, enunciados comprometidos, críticas y desacuerdos. En los siguientes apartados se desarrollarán dichos contextos para evidenciar que, en tales casos, la risa funciona como una estrategia para fortalecer o proteger la imagen del hablante frente a actos amenazantes de imagen.

Antes de iniciar, es preciso brindar una síntesis de la conversación. Esta se estructuró a partir de temas personales y académicos. En ambos casos, los participantes se apoyaron en rondas de historias cuyo objetivo era ejemplificar puntos similares de alguna experiencia. El tema de la zoofilia fue el más exitoso y su alusión fue recurrente a lo largo de toda la interacción. 
Miguel y Ernesto poseen un estilo conversacional muy participativo, cuyas características son: el habla rápida, los solapamientos, la presencia de temas personales, la toma de turnos rápida, la presencia de rondas de historias en las cuales el punto se dramatiza, entre otras. Un dato interesante sobre Miguel es que él es quien más utiliza continuadores del tipo «mh» para mostrar su interés hacia los relatos de sus amigos. Por su parte, Joaquín posee un estilo muy considerado en el cual respeta los turnos de habla de los interlocutores y, normalmente, prefiere el silencio frente a los solapamientos; su solidaridad hacia los demás hablantes se da mediante episodios de risa. A pesar de su estilo considerado, es quien introduce los temas más exitosos y que provocan risa.

\subsection{La risa en las impropiedades conversacionales}

La risa que aparece en este tipo de enunciados pretende cubrir posibles malentendidos, palabras obscenas o eufemismos (Cestero-Mancera, 1996). No obstante, en este trabajo también se considera pertinente incluir las palabras y temas tabúes y los enunciados con doble sentido. Por doble sentido se entiende el significado connotativo de algunos términos.

Una palabra tabú es aquella que no puede ser usada por el hablante sin causar una ofensa, pues se refiere a aspectos sexuales, religiosos o sobrenaturales; es un vocablo que no se pronuncia, por lo tanto, el hablante emplea un eufemismo, un "encubrimiento del significado" (Lewandowski, 1995, p. 128) o una expresión indirecta en lugar de una ofensiva, vergonzosa o desagradable (Crystal, 1999, p. 111). Brown y Levinson (1987) consideran los temas tabúes como actos amenazantes de imagen porque en ellos el hablante no muestra atención hacia la imagen positiva del oyente.

Uno de los temas más exitosos desarrollados durante la conversación es el de la zoofilia o sexo con animales. Este es presentado de manera anecdótica por parte de uno de los hablantes (Joaquín), quien narra lo escuchado por los lugareños mientras trabajaba en Mar de Oro, un pueblo alejado de la capital. En un punto de la interacción, los participantes conversan sobre la gran cantidad de licor ingerida por una persona, a quien se refieren como Svankovich. Joaquín comenta que mientras trabajaron juntos en el pueblo, había ocasiones en las cuales acudían a un bar para tomar y allí Svankovich se emborrachaba. Asimismo, en el comedor de la empresa les daban de comer «frito»: un plato en el cual se utilizan todas las vísceras del cerdo. En ese momento, Miguel y Ernesto molestan a Joaquín diciéndole que "toda la carne todos los animales que usted se comió se los cogieron" (líneas 1-2). Con esto se recuerda la historia de la zoofilia -introducido en los primeros minutos de la dinámica- y se revive así dicho tema tabú. Todo lo anterior desencadena en la intervención de Miguel en la línea 20 sobre otras preferencias sexuales de los lugareños y suscita una serie de episodios de risa.

a.

01 MIG: sí sí / todo toda la carne todos los animales que usted se comió se $02 \quad$ los cogieron

((5 líneas omitidas))

07 ERN: y Svankovich se los cogía [ pero después de muertos $\S$

((2 líneas omitidas))

10 MIG: después de muertos / ah sa- ah sí fijo porque entonces

((6 líneas omitidas))

$17 \quad$ MIG: con animales muertos $\S$ 


\begin{tabular}{|c|c|c|}
\hline 18 & JOA: & $\S$ ipor Dios! $\S$ \\
\hline 19 & MIG: & $\S$ y seguro de- haber debe \\
\hline 20 & & haber una perversión así como que se cogen plantas [ (Ra) \\
\hline 21 & ERN: & {$[(\mathbf{R a})$} \\
\hline 22 & JOA: & ¡ah sí! \\
\hline 23 & MIG: & ¿¡cómo que sí!? [ (Ra) \\
\hline 24 & JOA: & [ la / mae / sí sí las de plátano \\
\hline 25 & MIG: & (Ra) \\
\hline 26 & JOA: & las matas de plátano las de guineo las de cuadrado / no sé cómo \\
\hline 27 & & les dicen \\
\hline 28 & ERN: & ajá / sí § \\
\hline 2 & JOA: & $\S$ les meten les meten el machete $(\mathrm{NV}$ : dedo índice derecho \\
\hline
\end{tabular}

30 a la altura de la cara se introduce en un hueco imaginario) les hacen

31 como así y y lo meten ahí / eso nos contaron también mae

La intervención de Miguel en la línea 20 es impropia, pues la sola mención del término «coger» genera incomodidad al referirse directamente al tema tabú de la sexualidad (el acto sexual y una perversión). La tensión producida por lo dicho debe ser liberada y, en este caso específico, ello se efectúa mediante un episodio de risa emitido por Miguel al final de su enunciado y secundado por Ernesto en la línea 21.

El episodio de risa de la línea 20 presenta dos posibles interpretaciones: a) una impropiedad conversacional, lo cual se refuerza al analizarse tomando en cuenta su contexto situacional y co-texto lingüístico, que remiten a la impropiedad; y b) un apoyo a la intervención de Miguel (línea 20) porque sirve de toma de turno: intervención verbal de Miguel (línea 20), episodio de risa de Ernesto (línea 21) e intervención verbal de Joaquín (línea 22).

La línea 23 está formada por dos elementos: uno verbal (“¿jcómo que sí!?”) y otro no verbal (episodio de risa), y es producida después de que Joaquín ha confirmado la suposición de Miguel sobre los lugareños: "se cogen plantas" (línea 23). El asombro de Miguel es evidente en el tono de su pregunta (indicado, en la transcripción, mediante la inclusión de la pregunta entre signos de admiración y de interrogación a la vez) y lo acompaña con un episodio de risa cuyo fin es resaltar tal admiración y, al mismo tiempo, liberar la tensión generada por un tema tabú.

Joaquín, seguidamente, detalla la planta utilizada para tal práctica (líneas 26-27) y explica cómo se realiza (líneas 29-31). Justo después de escuchar el tipo de planta empleada, Miguel interviene en 25 con un episodio de risa que puede analizarse de dos formas: a) como una impropiedad conversacional: el contenido del enunciado precedente es impropio (es decir, se "cogen" las matas de plátano) y, por ende, la risa siguiente se contempla dentro de ese contenido; y b) como atenuación al contenido, atenuación que, al no haber sido efectuada por el relator, es realizada por el interlocutor como una forma de solidaridad con aquel.

\subsection{La risa en los enunciados comprometidos}

La risa también aparece en enunciados comprometidos, los cuales son entendidos como críticas culturales y sociales, expresiones sobre opiniones o gustos personales y enunciados irónicos o descorteses (Cestero-Mancera, 1996).

En el siguiente segmento, los participantes aceptan entre bromas estar a favor de la minería y de la exploración petrolera, pero ser incomprendidos por quienes defienden los 
recursos naturales. La posición sobre el aspecto de la naturaleza es bastante diferente entre los participantes y las personas aludidas en la conversación. Dentro de este marco de conflictos ecológicos y pensamientos disímiles, Joaquín introduce el tema de la mina: los lugareños y los ecologistas piensan que por el orificio cavado se saldrá el mar, el cianuro será utilizado para fabricar bombas atómicas y, por esa razón, Estados Unidos invadirá nuestro país. Ante tal panorama, la intervención de Ernesto es un enunciado comprometido (líneas 1-2).

b.

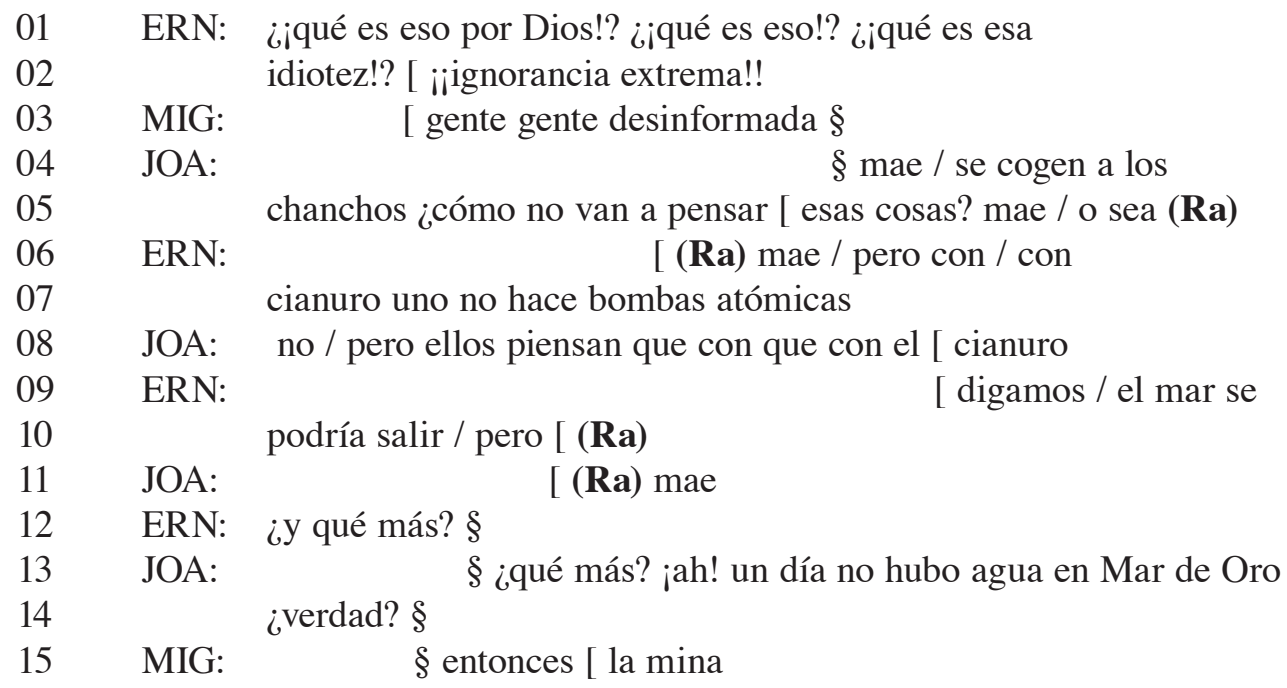

Las líneas 1 a 3 son un juicio directo hacia los pobladores de Mar de Oro, al ser considerados ignorantes o desinformados por pensar que la mina producirá los efectos arriba mencionados. A pesar de ser críticas negativas, se consideran como enunciados comprometidos porque no son dirigidas a una única persona, sino a un grupo y, además, su contenido es irónico y atenta contra la imagen de los aludidos.

Aunque las líneas 1-2 también son juicios directos, debe notarse la ausencia de risa, a pesar de que todos los hablantes participan en la elaboración de la crítica social. Si se toman tales enunciados como actos amenazantes de imagen (AAI), entonces los hablantes no están preocupados por evitarlos, mitigarlos o repararlos porque las personas citadas son ajenas a ellos y no les interesa defenderlas o alabarlas. Los lugareños son totalmente desconocidos para ellos $y$, por eso, no efectúan ninguna estrategia para salvar la imagen.

Por otro lado, los episodios de risa de las intervenciones 5-6 también podrían analizarse como impropiedad conversacional porque se hallan en un enunciado con una referencia sexual («se cogen a los chanchos»). Sobre este doble análisis, Bolaños (2010a) plantea que según el contexto de aparición de la risa, un mismo episodio puede poseer dos o más interpretaciones, lo cual evidencia que el estudio de la risa no excluye interpretaciones, sino, al contrario, se enriquece con otras.

Las líneas 10-11 presentan emisiones simultáneas de risa también interpretadas como enunciados comprometidos, ya que poseen un sentido irónico al afirmar que el mar podría salirse por el orificio cavado en la mina, pues los participantes saben de la improbabilidad de este hecho. Ernesto irrespeta la máxima griceana de calidad, pues proporciona información falsa (el mar no se saldrá por el orificio de la mina); esto implica que sus interlocutores deben comprender el enunciado como irónico y no considerarlo como un acto de habla con valor veritativo. 
Ahora bien, lo llamado como enunciados comprometidos por Cestero-Mancera (1996) son actos amenazantes de imagen (AAI). Por tal motivo, la interpretación de las emisiones de risa presentes en estos enunciados también deberán entenderse como una estrategia de cortesía. Esto implica que la risa, en este contexto, además de atenuar el contenido del enunciado mismo, funciona como una estrategia para salvar la imagen de los interlocutores.

\subsection{La risa en las críticas}

La risa aparece en las críticas negativas, del propio hablante o de una tercera persona, por defectos, costumbres o gustos (Cestero-Mancera, 1996) y su función es debilitar el contenido de la crítica. Sin embargo, para el caso específico del corpus de esta investigación, solo se hallaron episodios de risa donde se potencia el ataque contenido en el juicio. Brown y Levinson (1987) consideran las críticas como actos amenazantes de imagen (AAI) y las incluyen en la categoría de las expresiones de desaprobación, los insultos, las acusaciones y las burlas porque indican que el hablante tiene una evaluación negativa de algún aspecto de la imagen social de la persona. Si se considera el aporte de estos autores, entonces se comprenderán los episodios de risa hallados en críticas como estrategias para potenciar el ataque.

El siguiente segmento se estructura a partir de la narración de la zoofilia. Ernesto reproduce una conversación sostenida con una chica llamada Sofía, en la cual comentaban que una pareja de amigos, Lía y Svankovich, tenía prácticas sexuales muy particulares (líneas 117, 119 y 121-122).

\begin{tabular}{|c|c|c|}
\hline & c. & \\
\hline 01 & ERN: & entonces me dice Sofía fijo tiene que co-que coger miles \\
\hline 02 & MIG: & $\mathrm{mh}$ \\
\hline 03 & ERN: & entonces le digo yo / sí / pero pero pero pero unas varas grotescas \\
\hline 04 & & has- rarísimas hasta con animales $\S$ \\
\hline 05 & JOA: & $\S(\mathbf{R a})$ \\
\hline 06 & ERN: & decía Sofía sí / claro claro claro y ya / entonces decía \\
\hline 07 & & Sofía seguro que a Svankovich le gusta como como como que le \\
\hline 08 & & metan cosas también $\S$ \\
\hline 09 & JOA: & $\S(\mathbf{R a})$ \\
\hline 10 & MIG: & $\mathrm{mh} \S$ \\
\hline 11 & ERN: & $\S$ pero eso es generalizado ¿verdad? ¿ya? (NV: entre risas) \\
\hline 12 & JOA: & {$[(\mathbf{R a})$} \\
\hline 13 & ERN: & [ o sea / es que Svankovich y Lía $§$ \\
\hline 14 & MIG: & $\S$ es un sentimiento generalizado \\
\hline 15 & ERN: & 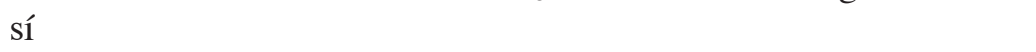 \\
\hline
\end{tabular}

Miguel participa en tres ocasiones, de forma verbal, con enunciados de apoyo: dos señales de retrocanalización (líneas 2 y 10) y con una frase eco (línea 14 es eco de la 11). En el caso de Joaquín, sus aportes son no verbales: en las líneas 5 y 9 se ríe justo después de escuchar los enunciados críticos emitidos por Ernesto contra Lía y Svankovich. Dichos aportes no verbales funcionan como una manera de mostrar su presencia en la conversación, es decir, como señales de retrocanalización. El estilo conversacional de Joaquín es muy considerado, por lo cual se comprende, entonces, que sus colaboraciones sean no verbales. 
Al ser la crítica referida a los gustos en cuanto a prácticas sexuales, le da un "tinte" de impropiedad conversacional al enunciado. Las emisiones de risa de las líneas 5 y 9 podrían también interpretarse como impropiedades conversacionales y se reforzaría, así, como la plantea Bolaños (2010a), el hecho de que una misma emisión de risa puede analizarse de varias formas sin que estas sean excluyentes.

Sobre el episodio de la línea 12 cabe destacar que es una risa de apoyo a la intervención de Ernesto (línea 11), quien verbaliza esa solicitud mediante el “iverdad? ¿ya?”. Joaquín le colabora de una manera no verbal, además de servir de seguimiento del mensaje.

Asimismo, podrían interpretarse todos los episodios de este ejemplo como: a) señales de retrocanalización emitidas por Joaquín para indicarle atención a Ernesto aunque no participe verbalmente en ese momento, y b) como un acto de solidaridad para mitigar el contenido tabú de los enunciados y liberar la tensión de este tipo de críticas impropias. Esto significa que la risa puede analizarse, al mismo tiempo, como una señal de retrocanalización y una estrategia de cortesía (al tratarse de un AAI).

Las críticas encontradas en este corpus son negativas y dirigidas a terceras personas, así que los episodios de risa podrían interpretarse no como un recurso para mitigar la crítica, sino, por el contrario, como una estrategia para potenciar la amenaza a la imagen de las personas criticadas y, al mismo tiempo, mostrar solidaridad con quien pronuncia la crítica. Sin embargo, tal análisis solo puede realizarse al considerar el estilo conversacional de los hablantes, el co-texto lingüístico y el contexto en el cual se desarrollan las críticas.

\subsection{La risa en los desacuerdos}

La risa aparece en enunciados que muestran desacuerdo con el enunciado precedente, en los que intentan restar importancia a los fallos del interlocutor o en las alabanzas explícitas (Cestero-Mancera 1996). En tales casos, la risa funciona como una atenuación. Brown y Levinson (1987) califican los desacuerdos como actos amenazantes de imagen (AAI) porque evidencian, eventualmente, que el hablante tiene una evaluación negativa de algún aspecto del interlocutor.

En el siguiente segmento (d.) los participantes conversan sobre la costumbre de los lugareños de Mar de Oro de practicar sexo con animales y con plantas. Joaquín relata el episodio en el cual uno de sus baquianos cuenta cómo es tener sexo con una mata de plátano, ante lo cual sus interlocutores parecen estar impresionados por tal conducta (líneas 1-2). Ernesto le pregunta a Joaquín si lo había intentado, pero este lo niega, seguido de una risa (línea 11).

d.

$01 \quad$ MIG: ¡ah mae! ¡qué impresionante!

02 ERN: mae mae ¿y sería cierto? $\$$

03 JOA: $\quad$ mae / diay los ma- el mae decía sí sí mae

$04 y$ como tiene una lechilla entonces es como pegajoso y se siente rico

$05 \quad$ y no sé qué $\S$

06 MIG:

07 ERN:

08 JOA:

09 nos estaba [ contan-

$\S[$ ¡iihh! mae

[ y él / o sea ¿él lo había hecho? §

10 ERN: $\quad[$ ¿y ustedes no lo intentaron? fijo $\S$ 


$\begin{array}{ccc}11 & \text { JOA: } & \text { § no [ (Ra) } \\ 12 & \text { MIG: } & \text { [ (Ra) } \\ 13 & \text { ERN: } & \text { [ (Ra) de la } \\ 14 & & \text { desesperación de estar ahí con los cuadrados / mae (NV: entre risas) } \\ 15 & \text { MIG: } & \text { no / entonces solo falta con rocas }\end{array}$

La risa de Joaquín en la línea 11 es un claro desacuerdo respecto al enunciado precedente sobre si intentó realizar tales prácticas sexuales. La estructura de su respuesta consta de un elemento verbal (la negación) seguido de uno no verbal (el episodio de risa que la refuerza) y, además, concuerda con su estilo conversacional muy considerado. Asimismo, tal risa sería una atenuación del desacuerdo.

El hecho de que los tres participantes sean amigos, los obliga a recurrir a mecanismos para salvar su imagen social con el fin de evitar conflictos entre ellos y la risa es uno de esos dispositivos. Tannen (2001) afirma que en ocasiones los hablantes evitan el desacuerdo y, con ello, aseguran el vínculo entre ellos, tal es el caso de las risas de Miguel y Ernesto (líneas 12-13). Ellos aceptan la invitación de Joaquín mediante la risa al final de su enunciado (línea 11) y así todos los participantes se convierten en cómplices.

El contexto en el cual se desarrolla este ejemplo es negativo porque se refiere a un tema tabú; en este caso, dichos episodios de risa pueden interpretarse como una impropiedad conversacional. Además, al ser un ataque a la imagen social de Joaquín y su defensa ante tal amenaza son una estrategia de cortesía.

Podría pensarse que los desacuerdos afectan negativamente el desarrollo de la interacción al indisponer o poner en contra a participantes y, entonces, llevar a la ruptura de la cooperación existente, según Grice (1998), en las conversaciones. No obstante, debe recordarse que se trata de una conversación entre amigos, quienes persiguen otros fines, por lo cual los desacuerdos tratan de evitarse. Para ello, los hablantes despliegan algunos recursos para mitigar los conflictos potenciales y la risa es uno de ellos.

Se esperaría que entre amigos los hablantes no se preocuparan por evitar los desacuerdos porque ellos comparten una relación social simétrica y una relación vivencial mutua. Piénsese por un momento en la relación entre hermanos, en la cual, en ocasiones, no se dan mitigaciones. Sin embargo, en este corpus específico no se observa que, frecuentemente, los participantes estén potenciando las amenazas.

Finalmente, al ser los desacuerdos considerados como AAI, entonces, la risa que se presenta en ellos debe, además, interpretarse como una estrategia de cortesía que mitigue las amenazas a la imagen pública de los interlocutores.

\section{Consideraciones finales}

La conversación analizada se estructuró a partir de dos temas principales: la zoofilia y las anécdotas de trabajo y estudio. Básicamente, ambos tópicos se presentaron a manera de rondas de historias en las cuales se dramatizó el punto de esta y fueron usadas para ilustrar experiencias similares. El primer tema correspondió a los enunciados impropios o negativos que generaron risa como una forma de mitigar el contenido de lo dicho, así como una manera de relajar la tensión corporal y social causada por un asunto tabú.

La conversación se considera como natural porque los hablantes se olvidaron de la presencia de la cámara de vídeo una vez pasados unos cuantos minutos; además, según Duranti 
(2000), los hablantes se las ingenian para conversar, aún con una cámara apuntándolos. Este hecho se confirma al hallarse muchos episodios de risa motivados por las impropiedades conversacionales y, también, porque algunas rondas de historias se referían abiertamente a temas tabúes; es decir, que los hablantes se olvidaron de la presencia de la cámara y hablaron sin inhibiciones.

En el caso específico de esta conversación, se encontraron enunciados que compartieron el común denominador de ser actos amenazantes de imagen (impropiedades conversacionales, enunciados comprometidos, críticas y desacuerdos). Como se pudo observar a lo largo del análisis, en esta conversación, la risa presente en enunciados considerados como actos amenazantes de imagen tiene como fin servir de estrategia de cortesía para reforzar la imagen pública del participante, amortiguar un eventual ataque o potenciar una amenaza a la imagen social.

\section{Notas}

1. En su habilidad de mostrar afiliación, amistad o aún intimidad, la risa juega un papel importante en la creación y mantenimiento de las relaciones interpersonales. (Nota aclaratoria: todas las traducciones han sido efectuadas por la investigadora).

2. El término "además" no pertenece al texto de Bravo.

3. Los corpora son naturales en el sentido de que no se hizo ningún intento por controlar cómo hablaba la gente o sobre qué hablaba en las grabaciones.

4. Estudios sugieren que es más probable que la gente se ría cuando los demás a su alrededor están riendo.

5. Generalmente una pregunta induce una respuesta.

6. La risa restaura y mantiene el vínculo a pesar de los choques en la interacción.

\section{Bibliografía}

Bachorowski, J. y Owren, M. J. (2004). Laughing Matters. Psychological Science Agenda. APA Online. 18 (9). http://www.apa.org/science/psa/sb-bachprt.html [Consulta 10 de noviembre de 2005].

Bolaños, A. (2007). La función de la risa en la conversación coloquial entre amigos. (Tesis de Maestría en Lingüística). Universidad de Costa Rica.

Bolaños, A. (2010a). La risa y el contexto. Revista Umbral.26 (1), 29-35.

Bolaños, A. (2010b). La risa: elemento regulador del flujo conversacional. Revista InterSedes. 11 (20), 22-35.

Bravo, D. (1996). La risa en el regateo: Estudio sobre el estilo conversacional de negociadores españoles y suecos. Stockholms Universitet: Institutionen för spanka och portugisiska. Edsbruck, Akademitryck.

Bravo, D. (1997). ¿Reírse juntos?: un estudio de las imágenes sociales de hablantes españoles, mexicanos y suecos. Diálogos Hispánicos. (22), 315-364.

Bravo, D. (2000). Risas y contrastes en los estilos comunicativos de negociadores españoles y mexicanos. Signo \& Seña. Revista del Instituto de Lingüística de la Facultad de Filosofía y Letras. (11), 133-165.

Briz, A. (1998). El español coloquial en la conversación. Esbozo de pragmagramática. Barcelona: Editorial Ariel. 
Brown, P. y Levinson, S. (1987). Politeness. Some universals in language usage. Cambridge: Cambridge University Press.

Carroll, D. (2002). Laughter in Conversation. http://homepage.mac.com/dcarroll2/2002/ JECA/lecture13.htm [Consulta 9 de enero de 2007].

Cestero-Mancera, A. M. (1996). Funciones de la risa en la conversación en lengua española. Lingüística Española Actual. 18 (2), 279-298.

Crystal, D. (1999). The Penguin Dictionary of Language. London: Penguin Books.

Devereux, P. G. y Ginsburg, G. P. (2001). Sociality Effects on the Productions of Laughter. Journal of General Psychology Online. 128 (2), 227-240.

Duranti, A. (2000). Antropología lingüística. Madrid: Cambridge University Press.

Glenn, P. (2003). Laughter in Interaction. Cambridge: Cambridge University Press.

Grice, H. P. (1998). Presuposición e implicatura conversacional. Por M. T. Julio y R. Muñoz (Comp.). Textos clásicos de pragmática. (105-124). Madrid: Arco Libros.

Lewandowski, T. (1995). Diccionario de lingüística. Marid: Ediciones Cátedra.

Provine, R. (1996). Laughter. http://www.americanscientist.org/template/AssetDetail/ assetid/24591/page [Consulta 10 de noviembre del 2005].

Robinson, D. y Smith-Lovin, L. (2001). Getting a Laugh: Gender, Status, and Humor in Task Discussions. Social Forces. 80 (1), 123-158.

Soilevuo-Grønnerød, J. (2004). On the meanings and uses of laughter in research interviews. Relationships between interviewed men and a woman interviewer. Young. Nordic Journal of Youth Research. 12 (1), 31-39.

Tannen, D. (2001). You Just Don't Understand. New York: Quill.

Tusón, A. (2002). El análisis de la conversación: entre la estructura y el sentido. Estudios de Sociolingüística. 3 (1), 133-153.

\section{Anexos}

Para la transcripción del corpus de este trabajo, se emplearon las convenciones propuestas por Briz (1982, pp. 13-14) en el proyecto del grupo Val.Es.Co. y que se reproducen a continuación:

A: $\quad$ Intervención de un hablante identificado como A.

¿?: $\quad$ Interlocutor no reconocido.

$\S$

Sucesión inmediata, sin pausa apreciable, entre dos emisiones de distintos hablantes.

Lugar donde inicia un solapamiento o superposición.

Reinicios y autointerrupciones sin pausa. 


\section{l

$\rightarrow$

(imperceptible)

$((\ldots))$

pa'l

$\mathrm{x}-\mathrm{y}-\mathrm{z}$

${ }^{\circ}()^{\circ}$

(Ra)

aaa

nnn

ii i?

i?

i !

Letra cursiva:

(AP: $\mathrm{xxxx})$

$(\mathrm{NV}: \mathrm{xxx})$
Silencio (pausa o intervalo) de cinco segundos; se especifica el número de segundos en las pausas de más de un segundo, cuando sea especialmente significativo.

Pausas naturales al hablar. ${ }^{1}$

Entonación ascendente o continuativa.

Entonación descendente.

Entonación mantenida o suspendida.

Fragmento indescifrable. ${ }^{2}$

Interrupciones de la grabación o de la transcripción.

Fenómenos de fonética sintáctica entre palabras, especialmente marcados.

Palabras o nombres deletreados.

Fragmento pronunciado en un tono de voz más bajo, próximo al susurro.

Significa "risas". 3

Alargamiento vocálico.

Alargamiento consonántico.

Preguntas o exclamaciones retóricas (por ejemplo, las interrogaciones exclamativas: preguntas que no preguntan).

Interrogaciones. También para los apéndices del tipo «¿no?, ¿eh?, ¿sabes?».

Exclamaciones. Expresiones irónicas.

Reproducción e imitación de emisores. Estilo directo, característico de los denominados relatos conversacionales.

Anotaciones pragmáticas que ofrecen información sobre las circunstancias de la enunciación. Rasgos complementarios del canal verbal. Añaden informaciones necesarias para la correcta interpretación de determinadas palabras (por ejemplo, la correspondencia extranjera de la palabra transcrita en el texto de acuerdo con la pronunciación real), etc. ${ }^{4}$

Anotaciones de elementos no verbales que indican tono de voz, mímica, gestos, ademanes, entre otros. ${ }^{5}$

\footnotetext{
1. Convención modificada por la investigadora.

2. Convención modificada por la investigadora.

3. El texto original de Briz (1998) presenta la palabra "risas" en mayúscula. Sin embargo, en este trabajo, los elementos no verbales se indicarán entre paréntesis y en minúscula, convención modificada por la investigadora.

4. Convención modificada por la investigadora.

5. Convención incluida por la investigadora.
} 